\title{
Front Matter: Volume 11076
}

, "Front Matter: Volume 11076," Proc. SPIE 11076, Advances in Microscopic Imaging II, 1107601 (3 October 2019); doi: 10.1117/12.2542433

SPIE. Event: European Conferences on Biomedical Optics, 2019, Munich, Germany 


\section{PROGRESS IN BIOMEDICAL OPTICS AND IMAGING}

\section{Advances in Microscopic Imaging II}

\section{Emmanuel Beaurepaire \\ Francesco Saverio Pavone \\ Editors}

\section{6-27 June 2019}

Munich, Germany

Sponsored by

The Optical Society (United States)

SPIE

Published by

SPIE 
The papers in this volume were part of the technical conference cited on the cover and title page. Papers were selected and subject to review by the editors and conference program committee. Some conference presentations may not be available for publication. Additional papers and presentation recordings may be available online in the SPIE Digital Library at SPIEDigitalLibrary.org.

The papers reflect the work and thoughts of the authors and are published herein as submitted. The publisher is not responsible for the validity of the information or for any outcomes resulting from reliance thereon.

Please use the following format to cite material from these proceedings:

Author(s), "Title of Paper," in Advances in Microscopic Imaging II, edited by Emmanuel Beaurepaire, Francesco Saverio Pavone, Proceedings of SPIE-OSA Vol. 11076 (SPIE, Bellingham, WA, 2019) Seven-digit Article CID Number.

ISSN: 1605-7422

ISSN: $2410-9045$ (electronic)

ISBN: 9781510628458

ISBN: 9781510628465 (electronic)

Copublished by

SPIE

P.O. Box 10, Bellingham, Washington $98227-0010$ USA

Telephone +1 3606763290 (Pacific Time) · Fax +1 3606471445

SPIE.org

and

The Optical Society

2010 Massachusetts Ave., N.W., Washington, D.C., 20036 USA

Telephone +1 202/223 8130 (Eastern Time) · Fax + 1 202/223 1096

http://www.osa.org

Copyright (C) 2019, Society of Photo-Optical Instrumentation Engineers and The Optical Society

Copying of material in this book for internal or personal use, or for the internal or personal use of specific clients, beyond the fair use provisions granted by the U.S. Copyright Law is authorized by SPIE subject to payment of copying fees. The Transactional Reporting Service base fee for this volume is $\$ 21.00$ per article (or portion thereof), which should be paid directly to the Copyright Clearance Center (CCC), 222 Rosewood Drive, Danvers, MA 01923. Payment may also be made electronically through CCC Online at copyright.com. Other copying for republication, resale, advertising or promotion, or any form of systematic or multiple reproduction of any material in this book is prohibited except with permission in writing from the publisher. The CCC fee code is $1605-7422 / 19 / \$ 21.00$.

Printed in the United States of America by Curran Associates, Inc., under license from SPIE.

Publication of record for individual papers is online in the SPIE Digital Library.

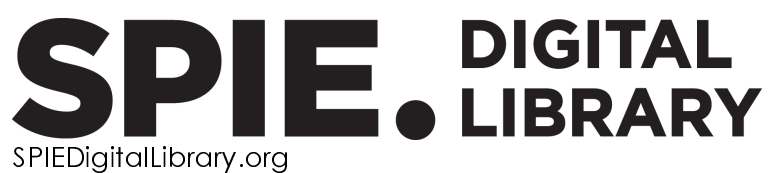

Paper Numbering: Proceedings of SPIE follow an e-First publication model. A unique citation identifier (CID) number is assigned to each article at the time of publication. Utilization of CIDs allows articles to be fully citable as soon as they are published online, and connects the same identifier to all online and print versions of the publication. SPIE uses a seven-digit CID article numbering system structured as follows:

- The first five digits correspond to the SPIE volume number.

- The last two digits indicate publication order within the volume using a Base 36 numbering system employing both numerals and letters. These two-number sets start with 00, 01, 02, 03, 04, 05, 06, 07, 08, 09, 0A , OB ... 0Z, followed by 10-1Z, 20-2Z, etc. The CID Number appears on each page of the manuscript. 


\section{Contents}

$\begin{array}{ll}\text { vii } & \text { Authors } \\ \text { xi } & \text { Conference Committee }\end{array}$

SESSION 1 SUPER-RESOLUTION IMAGING

$1107602 \quad$ Adaptive holographic region of interest illumination with oblique angles for use in single molecule localization microscopy (Invited Paper) [1 1076-1]

1107603 Robust online image processing for high-throughput super-resolution localization microscopy [11076-2]

1107605 Automated multiwell plate STORM: towards open source super-resolved high content analysis [11076-4]

1107606 Advances in microscopic imaging at the nanoscale using soft X-rays and extreme ultraviolet (EUV) from a compact laser plasma source [11076-6]

\section{SESSION 2 NEUROPHOTONICS}

$1107607 \quad$ Chromatic serial multiphoton microscopy for multicolor imaging of large brain volumes (Invited Paper) [1 1076-7]

1107608 Label-free imaging of bipolar cell axons in mouse retina by second-harmonic generation (Invited Paper) [1 1076-8]

1107609 Full-optical stimulation and readout of neuronal activity during optogenetically-evoked movements in awake mice [1 1076-9]

11076 OA Myeloarchitecture of the murine neocortex visualized by intrinsic third-harmonic generation [1 1076-10]

11076 OB Three-dimensional analysis of human brain cytoarchitectonics by means of a SWITCH/TDEcombined clearing method [1 1076-11]

11076 OC Deep learning strategies for scalable analysis of high-resolution brain imagery [1 1076-12]

11076 OE Label-free THG imaging of bone tissue microstructure: effect of low gravity on the lacuno-canalicular network [11076-14] 
11076 OF Correlative multiphoton microscopy and infrared nanospectroscopy of label-free collagen [1 1076-15]

11076 Ol Nonlinear imaging of female breast tissue biopsies [1 1076-18]

SESSION 4 EXCITATION SHAPING AND ADVANCED METHODS

11076 0J Compressive endo-microscopy (Invited Paper) [1 1076-19]

11076 OL Flat field illumination for improved fluorescence microscopy [1 1076-21]

11076 ON Light-sheet modulation for increased light-efficiency in a selective plane illumination microscope [1 1076-23]

$1107600 \quad$ High resolution 3D imaging of primary and secondary tumor spheroids using multicolor multiangle Light Sheet Fluorescence Microscopy (LSFM) [1 1076-24]

11076 OP Instantaneous isotropic volumetric imaging of fast biological processes [1 1076-77]

SESSION 5 QUANTITATIVE PHASE IMAGING: METHODS

11076 OS Wide field of view self-referencing quantitative phase contrast microscopy based on Lloyd's mirror interferometer (Invited Paper) [1 1076-29]

SESSION 6 QUANTITATIVE PHASE IMAGING: BASIC SCIENCE

11076 ОT Label-free detection of global morphology changes in confluent cell layers utilizing quantitative phase imaging with digital holographic microscopy (Invited Paper) [1 1076-30]

11076 OU Large scale high sensitivity optical diffraction tomography of zebrafish [1 1076-31]

11076 OV Blood cell - vessel wall interactions probed by reflection interference contrast microscopy [1 1076-32]

SESSION 7 QUANTITATIVE PHASE IMAGING: COMPUTATION

$110760 Z$ Label-free nanoscopy enabled by coherent imaging with photonic waveguides [1 1076-36]

1107610 Deep-learning for phase unwrapping in lens-free imaging [1 1076-37]

iv 
$1107611 \quad$ Three-dimensional nanoscale nuclear architecture mapping for improved cancer risk stratification (Invited Paper) [1 1076-38]

1107612 Optical biomarkers for detection of malignant tissue using digital holographic microscopy [1 1076-39]

\section{SESSION 9 POLARIZATION-RESOLVED IMAGING}

1107613 Fast P-THG microscopy for the characterization of biomaterials [1 1076-40]

1107614 Fast polarization imaging in coherent Raman scattering for the observation of lipid order dynamics in real-time [11076-41]

$1107615 \quad$ Mueller microscopy of full thickness skin models combined with image segmentation [1 1076-42]

1107616 Fast Mueller linear polarization modality at the usual rate of a laser scanning microscope [1 1076-43]

SESSION 10 ADVANCED APPLICATIONS

$1107618 \quad$ Multi-target immunofluorescence using spectral FLIM-FRET for separation of undesirable antibody cross-labeling [1 1076-45]

$1107619 \quad$ Label-free, scalable and point-of-care imaging platform for rapid analysis of biomarker [11076-46]

11076 1A Detection of trichomonal vaginalis through lensless optofluidic microscopy [1 1076-47]

11076 1B Microplastics detection and environmentally toxicity testing by multimodal optical metrology [1 1076-48]

\section{POSTER SESSION}

11076 1D Characterising cross-coupling in coherent fibre bundles [1 1076-50]

$110761 \mathrm{E} \quad$ Piezo-fiber multipattern scanner for endoscopy [1 1076-51]

1107616 A spatiotemporal analysis using second harmonic generation reveals enhancement of type I collagen polymerization by dermatan sulfate glycosaminoglycans [1 1076-53] 
$110761 \mathrm{H} \quad$ Extended field-of-view microendoscopy through aberration corrected GRIN lenses [1 1076-54]

$11076 \mathrm{lJ} \quad$ Integration of highly-refractive spheres in microfluidic chips for high-contrast detection of bacteria with low-magnification systems [1 1076-56]

$110761 \mathrm{~K} \quad$ Probing chemosensitivity and energy metabolism in patients-derived colorectal cancer cells [1 1076-57]

$110761 \mathrm{~N} \quad$ Wavelet leader based multifractal analysis of phase contrast images for cervical pre-cancer detection [1 1076-60]

1107610 Phase transition monitoring in adipose tissue by multiphoton microscope [1 1076-61]

11076 1P Time encoded chromatic confocal microscopy for wide field 3D surface profiling [1 1076-62]

$110761 Q \quad$ Simulation approach to optimize fluorescence imaging performance of wide-field temporalfocusing microscopy with tunable wavelength excitation [1 1 1076-63]

11076 IR The potential of laser interferometry for a non-invasive assessment of biopolymer film structure and biological properties [1 1076-66]

11076 is Method for non-invasive hemoglobin oxygen saturation measurement using broadband light source and color filters [1 1076-67]

$110761 \mathrm{~T} \quad$ Diagnostic decision support tool for anemias based on label-free holographic imaging [1 1076-68]

$110761 \mathrm{U} \quad$ Holographic imaging for 3D cells morphology in microfluidic flow [1 1076-69]

11076 1W Adaptive fluctuation imaging captures rapid subcellular dynamics [1 1076-72]

$110761 \times \quad$ Response of patient-specific cell cultures to photodynamic treatment analyzed by digital holographic microscopy [1 1076-73]

11076 1Y Concept and optical design of the underwater microscope [11076-74]

1107612 Real-time visualization of structural and biochemical information using single laser source [1 1076-75]

1107620 Development of all-optical imaging system for studying cerebral blood flow regulation using optogenetics [1 1076-76]

$1107621 \quad$ Two-photon excited polarized fluorescence in NADH in methanol/water solutions [1 1076-79] 


\section{Authors}

Numbers in the index correspond to the last two digits of the seven-digit citation identifier (CID) article numbering system used in Proceedings of SPIE. The first five digits reflect the volume number. Base 36 numbering is employed for the last two digits and indicates the order of articles within the volume. Numbers start with 00, 01, 02, 03, 04, 05, 06, 07, 08, 09, 0A, OB...0Z, followed by 10-12, 20-2Z, etc.

Abdeladim, Lamiae, 07 Agarwal, Krishna, 1W Agelaki, Sofia, Ol

Ahluwalia, Balpreet S., OZ, IW

Aivalioti, Chrysoula, 00

Alexandrov, Y., 05

Allegra Mascaro, Anna Letizia, 09

Allier, C., 10

Altmann, Yoann, 1D

Altug, Hatice, 19

Amitonova, Lyubov V., OJ

Anand, Arun, OS

Andolfo, Immacolata, $1 T$

Antonini, Andrea, $1 \mathrm{H}$

Arganda-Carreras, Ignacio, 07

Athanassakis, Irene, $\mathrm{Ol}$

Avdonkina, N. A., 1X

Baker, J. R., 05

Balázs, Bálint, OP

Baldueva, I. A., 1X

Balla, Naveen Kumar, 14

Baranova, Natalia S., OV

Barnes, P. J., 05

Barroso, Álvaro, 1B

Bartnik, Andrzej, 06

Beaurepaire, Emmanuel, 07, 0E, 13

Becker, Jan, 02

Belashov, A. V., $1 \mathrm{X}$

Beltukov, Y. M., 21

Belushkin, Alexander A., 19

Bemelmans, Alexis-Pierre, 07

Bertoncini, Andrea, $1 \mathrm{H}$

Bezverkhnii, N. O., 21

Bhat, Ramray, $1 \mathrm{G}$

Bianco, Vittorio, $1 T$

Birgisdottir, Åsa B., 1W

Biswas, Rabindra, $1 G$

Bodermann, Bernd, is

Boutillon, Arthur, 13

Bovasianos, Savvas, Ol

Bovetti, Serena, $1 \mathrm{H}$

Bradu, Adrian, 16

Brasselet, Sophie, 14

Brehar, Felix, 12

Bucinca-Cupallari, Festa, 08

Bureau, Lionel, OV

Butmalai, Dan, 12

Calin, Violeta L., 12

Calvo-Lozano, Olalla, 19
Chessel, Anatole, 07

Chhnaiwal, Vani, OS

Chien, Fan-Ching, $1 Q$

Cioni, O., 10

Clavreul, Solène, 07

Coche-Guérente, Liliane, OV

Comanescu, Brindus, 12

Costantini, Irene, OB

Costea, Radu V., 12

Croop, B., OL

Danilova, A. B., $1 \mathrm{X}$

Davies, Heather S., OV

Dazzi, Alexandre, OF

de Boer, Johannes F., OJ

de Medeiros, Gustavo, OP

De Vito, Giuseppe, 09

Débarre, Delphine, $\mathrm{OE}, \mathrm{OV}$

Dembski, Sofia, 15

Deniset-Besseau, Ariane, OF

Dey, Priyanka, 19

Dhaliwal, Kevin, 1D

Donnelly, L. E., 05

Döpker, Eva, OT

Druzhkova, Irina, 1K

Dubrevil, Matthieu, 16

Ducourthial, Guillaume, 13

Dudenkova, Varvara, $1 \mathrm{~K}$

Duempelmann, LUC, 19

Dullo, Firehun T., OZ

Dumitru, Adrian, 12

Dunsby, C., 05

Dziomba, Thorsten, $1 \mathrm{~S}$

El Amri, Nouha, OV

Estevez, M.-Carmen, 19

Fàbrega, Anna, 19

Fabri-Faja, Nuria, 19

Fang, Qiyin, 1A

Fellin, Tommaso, $1 \mathrm{H}$

Ferhanoglu, Onur, $1 \mathrm{E}$

Ferraro, Pietro, 1T, $1 \mathrm{U}$

Fiedorowicz, Henryk, 06

Filippidis, George, ㅇ

Forli, Angelo, $1 \mathrm{H}$

Förster, Ronny, 02

Frasconi, Paolo, OC

French, P. M. W., 05

Frolov, Alexey D., 1Y

Frolov, Dmitry N., $1 Y$

Fuchs, U., OL 
Gambale, Antonella, IT

Garbellotto, Chiara, ON

Garcia, E., 05

Garcia-Caurel, Enric, 15

Gavgiotaki, Evangelia, OI

Gelfond, M. L., 1X

Genthial, Rachel, OE

Georgoulias, Vassilis, Ol

Gerbaix, Maude, OE

Gierten, Jakob, OP

Gijs, M. A. M., 1J

González-López, Juan J., 19

Gorbunova, I. A., 21

Görlitz, F., 05

Gourrier, Aurélien, OE

Graß, Stefan, 1B

Gregor, Ingo, 18

Grigore, Viorela N., 12

Groeber-Becker, Florian Kai, 15

Gweon, Bomi, 12

Han, K.Y., OL

Heintzmann, Rainer, 02

Hervé, L., 10

Hinze, Peter, 15

Hocke, Andreas C., 18

Hofer, Matthias, 14

Hufnagel, Lars, OP

Ignatova, Nadezhda, 1K

Iolascon, Achille, $1 T$

Jahani, Yasaman, 19

Jang, Hansol, 1P

Javidi, Bahram, OS

Jha, Keshav Kumar, 1G

Joglekar, Mugdha, OS

Jügler, Alexander, 02

Kaiser, Mathias, OT

Kalkman, Jeroen, $\mathrm{OU}$

Kalstad, Trine, IW

Kaminski, Clemens F., $\mathrm{OZ}$

Kang, Ungyo, 12

Karam-Eldaly, Ahmed, 1D

Kemper, Björn, OT, 1B

Ketelhut, Steffi, OT, 1B

Khaw, I., OL

Khayatzadeh, Ramin, $1 \mathrm{E}$

Kil'deeva, Nataliya, 1R

Kim, Chang-Seok, $1 \mathrm{P}$

Kim, Jin Won, 12

Kim, Minkyung, 20

Kiselev, Nikolay, $1 \mathrm{~K}$

Kistenev, Yuri V., 10

Koberling, Felix, 18

Kochubey, Vyacheslav I., 10

Krämer, Benedikt, 18

Kroker, Stefanie, 1S

Kumar, S., 05

Kun, Jessica, $1 \mathrm{~A}$

Latour, Gaël, OF

Laurino, Annunziatina, OB

Lazzeri, Erica, OB
Le Grand, Yann, 16

Lechuga, Laura M., 19

Lee, Hee Ryung, 15

Legouis, Renaud, 13

Leitgeb, Rainer, OS

Liapis, Evangelos, 00

Liberale, Carlo, $1 \mathrm{H}$

Lichtman, Jeff W., 07

Lightley, J., 05

Lim, Hyungsik, 08, 0A

Lisievici, Mihai, 12

Liv, Yang, 03, 11

Livet, Jean, 07

Lołz, Christian, 15

Loulier, Karine, 07

Lukina, Maria, 1K

Ma, Hongqiang, 03

Maffettone, Pier Luca, 14

Mahajan, Swapnil, OS

Mahou, Pierre, 07

Maldonado, Sebastián, IW

Mannelli, llaria, 19

Matho, Katherine S., 07

Mathurin, Jérémie, OF

Mazzamuto, Giacomo, OB, OC

McLaughlin, Stephen, 1D

Memmolo, Pasquale, 1T, $1 \mathrm{U}$

Menneteau, M., 10

Merola, Francesco, 1T, 1U

Metelin, Vladislav, $1 \mathrm{R}$

Miccio, Lisa, 1T, $1 \mathrm{U}$

Migliozzi, D., $1 \mathrm{~J}$

Mihailescu, Mona, 12

Moehl, A., OL

Moisescu, Mihaela G., 12

Montagni, Elena, 09

Morales, S., 10

Moretti, Claudio, $1 \mathrm{H}$

Morizet, Joséphine, 13

Mosser, Gervaise, OF

Mugnano, Martina, 1T, $1 \mathrm{U}$

Munro, I., 05

Myrmel, Truls, 1W

Navarro, F., 10

Neil, M. A. A., 05

Nekhaeva, T. L., $1 X$

Neri, Mattia, OB

Nikolaev, Viktor $\vee ., 10$

Norlin, Nils, OP

Novikova, Tatiana, 15

Olivier, Cécile, OE

Opstad, Ida S., 0Z, 1W

Oraiopoulou, Mariam-Eleni, 00

Orsini, Francesco, OC

Ossikovski, Razvigor, 15

Papamatheakis, Joseph, 00

Park, Se Jin, 1P

Parker, Helen E., 1D

Patrascu, Oana-Maria, 12

Pavone, Francesco Saverio, 09, OB, OC 
Pedrini, Giancarlo, OS

Pello, Josselin, 19

Perperidis, Antonios, 1D

Petrescu, George, 12

Peyrin, Françoise, $\mathrm{OE}$

Pohl, Luisa, OT

Pradhan, Asima, 1N

Prevedel, Robert, OP

Pruneri, Valerio, 19

Psycharakis, Stylianos E., 00

Raghunathan, Varun, $1 \mathrm{G}$

Rajamanickam, Vijayakumar P., $1 \mathrm{H}$

Redlich, Michael, OA

Reiber, Jens, 1B

Resta, Francesco, 09

Richter, Ralf P., OV

Ripoll, Jorge, 00

Rivet, Sylvain, 16

Roffilli, Matteo, OB, OC

Rohilla, Sumeet, 18

Rubayo-Soneira, J., 21

Russo, Roberta, $1 \mathrm{~T}$

Ryu, Jiheun, 12

Sacconi, Leonardo, $\mathrm{OB}$

Sahoo, Gyana Ranjan, 1N

Sakkalis, Vangelis, 00

Sarkar, Purba, $1 \mathrm{G}$

Sasin, M. E., 21

Sattin, Andrea, $1 \mathrm{H}$

Savopol, Tudor, 12

Sazhnev, Nikita, $1 R$

Scaglione, Alessandro, 09

Schanne-Klein, Marie-Claire, OE, OF, 13

Schnekenburger, Jürgen, OT, 1B

Semenova, I. V., $1 \mathrm{X}$

Shikhina, Nina, $1 \mathrm{R}$

Shimolina, Lyubov', 1K

Shin, Hyun-joon, 20

Shirmanova, Marina, $1 \mathrm{~K}$

Shukla, Shivam, iN

Silvestri, Ludovico, OB, OC

Simonetto, Andrea, $\mathrm{OB}$

Sintes, Jean-Marc, 07

Smieja, Marek, 1A

Soleymani, Leyla, 1A

Solinas, Xavier, 07

Song, Joon Woo, $1 \mathrm{Z}$

Stapelfeldt, Finn-Niclas, 15

Stringari, Chiara, 13

Ströhl, Florian, OZ, IW

Succol, Francesca, $1 \mathrm{H}$

Supatto, Willy, 07, 13

Tabachkov, Alexey G., 1Y

Tang, J., OL

Tanner, Michael G., 1D

Taylor, Jonathan M., ON

Tekpinar, Miyase, $1 \mathrm{E}$

Terborg, Roland A., 19

Thomson, Robert R., 1D

Tinguely, Jean-Claude, $\mathrm{OZ}$
Trivedi, Vismay, OS

Tsafas, Vassilis, 0

Tuchin, Valery V., 10

Turney, Steve, 07

Tzardi, Maria, OI

Uttam, Shikhar, 11

van Rooij, Jos, OU

Vasilenko, Irina, 1R

Vasyutinskii, O. S., 1X, 21

Verdier, Claude, OV

Vico, Laurence, OE

Villone, Massimiliano, $1 \mathrm{U}$

Vinogradova, Olga A., 1Y

Viri, V., 1J

Waag, Andreas, 1S

Wachulak, Przemysław, 06

Wagner, Nils, OP

Wasisto, Hutomo Suryo, 15

Weimann, Thomas, $1 \mathrm{~S}$

Wittbrodt, Joachim, OP

Wu, Wenze, 15

Wysoczanski, R., 05

$\mathrm{XU}$, Jianquan, 03

Yan, M., 05

Yanina, Irina Yu., 10

Yesilkoy, Filiz, 19

Yoo, Hongki, 12

Zacharakis, Giannis, 00

Zacharopoulos, Athanasios, 00

Zagainov, Vladimir, 1K

Zagaynova, Elena, $1 \mathrm{~K}$

Zakharova, Vasilina, 1R

Zhikhoreva, A. A., 1X, 21 
Proc. of SPIE Vol. 11076 1107601-10

Downloaded From: https://www.spiedigitallibrary.org/conference-proceedings-of-spie on 25 Apr 2023 Terms of Use: https://www.spiedigitallibrary.org/terms-of-use 


\title{
Conference Committee
}

\author{
General Chair \\ Rainer Leitgeb, Medizinische Universität Wien (Austria) \\ Programme Chairs
}

Brett E. Bouma, Wellman Center for Photomedicine (United States)

Paola Taroni, Politecnico di Milano (Italy)

Conference Chairs

Emmanuel Beaurepaire, Ecole Polytechnique, CNRS (France)

Francesco Saverio Pavone, LENS - Laboratorio Europeo di Spettroscopie Non-Lineari (Italy)

Conference Programme Committee

Paul J. Campagnola, University of Wisconsin-Madison (United States) Laurent Cognet, Université de Bordeaux (France)

Vincent R. Daria, The Australian National University (Australia)

Valentina Emiliani, Laboratory de Neurophysiologie et Nouvelles Microscopies (France)

Paul M. W. French, Imperial College London (United Kingdom) Irene Georgakoudi, Tufts University (United States)

Rainer Heintzmann, Leibniz-Institut für Photonische Technologien e.V. (Germany)

Jan Huisken, Morgridge Institute for Research (Germany)

U. Valentin Nägerl, Université de Bordeaux (France)

Jerome Mertz, Boston University (United States)

Nozomi Nishimura, Cornell University (United States)

Dan Oron, Weizmann Institute of Science (Israel)

Shy Shoham, NYU Langone Health (Israel)

Peter T. C. So, Massachusetts Institute of Technology (United States)

Vinod Subramaniam, Vrije University Amsterdam (Netherlands)

Ivo Vanzetta, Institut de Neurosciences de la Timone (France)

Alipasha Vaziri, The Rockefeller University (United States)

\section{Session Chairs}

1 Super-Resolution Imaging

Francesco Saverio Pavone, LENS - Laboratorio Europeo di

Spettroscopie Non-Lineari (Italy) 
2 Neurophotonics

Hervé Rigneault, Institut Fresnel (France)

3 Multiphoton Microscopy

Hyungsik Lim, Hunter College (United States)

4 Excitation Shaping and Advanced Methods

Gaël Latour, Université Paris-Sud (France)

5 Quantitative Phase Imaging: Methods

Gabriel Popescu, University of Illinois (United States)

6 Quantitative Phase Imaging: Basic Science

Yang Liu, University of Pittsburgh (United States)

7 Quantitative Phase Imaging: Computation

Pietro Ferraro, Istituto di Scienze Applicate e Sistemi Intelligenti "Eduardo Caianiello" (Italy)

8 Quantitative Phase Imaging: Clinical Applications

Renjie Zhou, The Chinese University of Hong Kong (Hong Kong, China)

9 Polarization-Resolved Imaging

Thomas Juffmann, Universität Wien (Austria)

10 Advanced Applications

Emmanuel Beaurepaire, Ecole Polytechnique, CNRS (France) 